\title{
Imbalance and Countermeasures -Thoughts on Biased Value Orientation of Higher Education in China and Corresponding
}

\author{
Adjustment \\ Daoxun Wang \\ Huanghe Science and Technology College, Zhengzhou, 450063, \\ China
}

\begin{abstract}
: the traditional framework of higher education laid particular stress on the superficial values of teaching tools and instructed students with monotonous methods, which neglected the improvement of humanistic quality and ignored the particular human-oriented values. The profession-oriented framework of the higher education should be necessarily changed. The newly established framework should focus on the contradiction mediation to highlight the unique value orientation. Include emotional education into the set system, only by which the original imbalanced state could be adjusted and the value orientation could be clearly defined.
\end{abstract}

Key words: imbalance and countermeasures; higher education; biased value orientation; adjustment

The imbalanced higher education only focused on the value of teaching tools, while failed to connect to the education of science and technology as well as humanistic education. The subjects relating to science and technology generally accounted for more proportion, resulting in imbalanced education in humanistic spirit. Reflecting on this kind of state should be started from the source to think about the integration. The education on spirit should be focused on the improvement of literacy level and the remodeling spirit, as well as strengthening the diverse cultural connections. Guidance on the college students should be focused on the sources, only by which the biases could be adjusted properly and the feasible adjustment system could be established. 


\section{General orientation bias}

The higher education that laid particular stress on value orientation was closely connected to daily practice and highlighted the interaction. The reform on the framework of the higher education had been suffering many obstacles, such as the constraint from the old system. Besides, the biased value orientation also limited the reform progress.

The specific value orientation of the higher education can be divided into macro and micro categories. To be specific, the source orientation of the macro level represented the overall tendency of the subsystems, which highlighted the performance appraisal of the cognition and a certain outstanding tendency of thoughts. The value appraisal of the macro level was the general cognitive appraisal and closely involved with the particular multi-decisions of higher education and daily actions. The value orientation of the micro level represented the multi-factors of the internal framework as well as the overall tendency. In the daily instruction, the micro multi-value highlighted the particular guidance. For example, there are differences between the selected methods for teaching activities.

The true orientation of the micro framework is closely connected with the daily teaching activities. Difficulties and biases in the two levels should be taken into consideration to recognize the bias of the orientation.

\section{Biases in the macro framework}

The overall orientation of the macro level contained hidden multiple biases. The values involving the higher education can be divided into internal direct value and indirect corresponding value. The internal direct value represented the noumenal value of education, highlighting the direct features. The indirect value represented correlated activities, which created multiple values, with clear features of tools. The traditional higher education was influenced by the hidden bias of Confucianism, in which the ethical value of higher education was regarded as prepared cognitive tools. The motivation of this kind of value produced unique utilitarianism of the educational connotation. Under the influence of general official standard, teachers and students took the prepared test scores as the measurement of appraisal. Studying decides one's 
future pushed teachers and students to focus on the improvement of their scores. However, if the education is irrelevant to one's scholarly honor or official rank, thus people will think that education is free of true value, resulting distorted tendency. Cultivating outstanding students through higher education was the only stipulated measure. The distorted cognition under the exam-oriented education pushed the particular distorted progress of higher education.

\section{Biases in the micro framework}

Micro cognitive bias was represented in the traditional authorities. People generally focused on traditional and stuck up for the authorities. This kind of cognition was significantly advantageous but impeded the development of the higher education in the new stage, resulting in difficulties. The long-term formed uniform textbooks and the prepared annual plans emphasized the consistency of the integrated state, by which the distinctive teaching framework was established. All colleges and departments in universities failed to follow their own judgment to arrange effective teaching activities.

The classes given and specific teaching correlation overlooked students' cognition. The selected teaching process generally adopted monotonous problem solving without leaving time for discussion. The prepared teaching plan failed to develop properly according to the investigated actual situation. The teachers failed to evaluate students' performance scientifically and objectively. The mechanical expounding failed to lay a foundation for frank interaction. Behind the superficial connection was the distorted relationship between teacher and students: failed to establish fair relationship, instead, teachers were in the absolute authoritative position and controlled students. The conservative value tendency was included in this kind of tendency particularly.

Generally, the value judgments of the macro and micro levels were of obviously fundamental properties. The macro orientation constrained the progress of daily instruction. The psychological progress of the young students was constrained by the authority, reflecting unfavorable tendency. Students were prone to feel inferior to their teachers, resulting unfair participation. There were short of necessary emotional communication between teachers and student, thus teachers can not meet students' 
potential demands. Therefore, the cultivated talents were short of necessary enthusiasm, innovation ability and independent cognition. The new cognition that the masters were supposed to own highlighted the shortage of the overall tendency.

\section{Novel thoughts on adjusting the complying standards}

The conservative orientation of the higher education impeded the progress of practice. Setting novel orientation is an inevitable trend, which provided good opportunity for following changes. In the new stage of knowledge-based economy, the bias reform involving the higher education possessed prepared material foundation. The unique replacement of the cognition provided with stable basement on thoughts. With the increasing unique cognition of the subjects, students gradually found their subject positions, which provided with internal motivation with potential features. Under this motivation, novel overall tendency should be created.

The established orientations in the specific field of higher education should be able to improve the development of the long-term system. It should prepare the complete system within the life-time cognition framework and regard practice as the starting point of the sources. The life-time education represented the social framework specific to studying, also represented the social orientation specific to modern features. It should be able to discover people's status, exploit students' potential, and reinforce the unique development under the diverse situations. The overall progress covers divided language skills, cognition skills under the wisdom framework, selected proper cognition method, action skills and psychology during cognizing.

\section{Specific adjustment methods}

i. Integration within the spirit framework.

The source spirit proposed by the higher education should be deliberately reconstructed. The reconstruction under the method of the spirit integration included the value transformation of the source. The changes in value of the source of the higher education didn't deviate from the prepared multiple functions. Focusing on the subjects should realize the various difficulties in the process of the development. Values of the fundamental tools and high-level spirit guidance should be integrated, by which the specific overall spirit can be integrated and this kind of spirit can be 
reconstructed.

The fundamental guidance established by the higher education should target to the lead students towards the future development and cultivate senior talents. Every subject receiving education should accept good education. Only by giving cognition goals with uniqueness can the new subjects with criticism awareness can be cultivated; and excellent self-examination psychology and sustainable source guidance can be established. To be specific, subjects relating to natural science should aim to cultivate high-level cognition skills and reliable source skills. Meanwhile, it should focus on setting the cultivation goals of spiritual character. There should be order and balanced emotional cognition in the higher levels. Similarly, the humanities should provide with applicable general knowledge and offer necessary space for spirit development. The prepared source goal of the humanities is the exclusive spiritual level of balance, creating best remedy value.

\section{ii. Emphasizing mutual connections among subjects}

Framework should be established to connect social sciences and related humanities. In the modern stage, the exclusive interaction among sci-tech economies represented the complementation between two kinds of subjects. The science subjects of the humanities discovered the differences between cognitions by relying on the set different perspectives. They have their own focuses in investigations and surveys, usual method of thinking and habits of adopting ways. It is impossible to combine subjects under the absolute state, however, the fundamental spirit of different subjects possess common features. The relying cognition and selected communication method should be integrated as well. Separating subjects under the dual framework means to separate the complete framework of the higher education. The integration of the higher education covered the connections among different cultures, based on which exclusive spirit integrating fields should be created. Culture that accepted by people should be mutually connected to highlight the clear effectiveness. It should avoid overlaying of the subjects on the same method, and focus on the management of the preset subjects, realizing integration of high levels. Cultural connection and integration should be obtained from the campus spirit of the sources. Do not place a 
set subject on other specific subjects. Facilitating the development of the subjects in the balanced state is of emphasized value.

iii. Highlight distinctive features

The future society will be a new society of extremely fierce competition. The technology development should be relied on the novel talents of innovative ability. Continuous creativity is the key to sustainable development. The cultivated talents should possess high-level innovation cognition. The existing framework of the higher education hasn't completely eliminated the common conservative and monotonous properties yet. It is necessary to emphasize diversity and individuality, and cultivate distinctive responsibility cognition and differentiate fundamental orientation. Researching distinctive and clear adjustment methods is related to preset orders. The novel methods followed the advantages of the old and facilitated the confirmation of the independent framework.

One of the characteristics of the information society is the multiple personalities under the diversification trend. All college students should be provided with opportunities of long-term development. They should be cultivated with distinctive exploiting cognition and complete personality. For example, subjects relating to psychology should cover the teaching contents: teach students to master their own life instead of being controlled arbitrarily; know how to interact and respect others; establish optimum self-discipline cognition; help them to form challenge cognition and long-term dream based on their original spirit status. Therefore, they can handle it when they suffer setbacks in their daily life and tolerate the sense of loss and boost their spirit again. Only by continuous innovation can they pursue novel matters.

iv. Specific changes in lives

The guidance on lives that provided by higher education should eliminate the traditional cognition and the cognition of the high-level authorities, thus the new framework of fair communication between teachers and students management theory of details could be determined. It should eliminate teachers' authoritative sense and focus on students' subject status as well as pay attention to cultivate independence and gradual creation of cognition. The selected teaching methods should be free of 
monotonous problem solving methods; novel methods of flexible framework should be established. The set appraisal indexes should include objective and diverse indexes. Do not take the test scores as the only appraisal standard. Ideological cognition should be established on the base of fair communication. Teachers should care about the students sincerely and make the preset ideological education routine and detailed. For example, communicate with students in their dormitories after class and solve their problems in study and life, providing basis for ideological guidance. During ideological interaction, democratic management should be adopted to motivate their initiative. A new teaching plan should be adopted after democratic discussion. Therefore, the college students will really fell that: they are the masters of the education; they can follow their own thoughts and ideas.

\section{v. Receive practical guidance}

The teaching programs set in the textbooks can be used for daily guidance. Only the practical cognition can be emphasized. The exclusive value of practice requires the students not following the textbooks and receiving the special classes provided by society. Expanding students' thoughts and horizon should not only be limited to examinations. The set appraisal system of the higher education should be changed by relying on the cognitive value of practice. Exam-oriented indexes should be reduced, while new indexes relating to practice should be increased. For example, students' cognitive psychology, performance of receiving new knowledge, ordinary inventions should be included into the appraisal indexes.

It should pay attention to that compared with the daily instruction in primary school; the higher education is much closer to life. It is because that the students shall enter society after graduated from colleges. At that time, the working experience and life experience that accumulated by the students will be put into use. Thus the cognition should be passed down to the students in the daily teaching tasks. Only by this, students can be free of confusion and solve the problem in the real life after enter society.

\section{Conclusion}

The value orientation of education is hidden in the prepared system which is a 
depth-level connected factor. Its hidden way influenced the behavior judgment of all stages and thoughts tendency. The long-term reform of the higher education reflected the difficulties which were produced by the conventional thoughts bias. Adapting to the changeable times should eliminate the conservative thoughts and seek maximum values. Reflections and adjustment on bias should be integrated with macro and micro levels, focus on adjusting details and exert real effects.

\section{Acknowledgments}

This paper is the phased objective of the Soft Science Research Program of the Science and Technology Department of Henan Province-“Strengthening and Innovation Research on Social Management of Henan Province” Series No.: 142400410172.

\section{Bibliography:}

[1] Zhang Heling. Reform on Value Orientation of the Higher Education in china [J]. Journal of Henan Institute of Education (Philosophy and Social Science Edition), $2011(01)$.

[2] Huang Jing. Thoughts on the Assessment Value of the Higher Education in China [J]. Seeker, 2014 (08)

[3] Dong Zefang, Huang Jianxiong. Review and Thoughts on the Changes of the Value Orientation of the Higher Education in China within the 60 Years. [J]. Journal of the Central China Normal University (Humanistic and Social Science Edition) 2011 (01). [4] Yuan Fang. The Value Orientation of the Higher Education under the Vision of the Ecological Civilization [J]. Social Sciences in Ningxia, 2013 (03).

[5] Zhang Yating. Discussion on the Value Orientation of the Higher Educational Philosophy [J]. Journal of Shanxi University of Finance and Economics, 2014 (S1) 